\title{
Operational Vibration-Based Response Estimation for Offshore Wind Lattice Structures
}

\author{
P. van der Male and E. Lourens \\ Department of Hydraulic Engineering, Faculty of Civil Engineering and Geosciences, \\ Delft University of Technology, Stevinweg 12628 CN Delft, the Netherlands \\ E-mail: p.vandermale@tudelft.nl
}

\begin{abstract}
The design for fatigue for offshore wind turbine structures is characterized by uncertainty, resulting from both loading specifications and numerical modelling. At the same time, fatigue is a main design driver for this type of structures. This study presents a strategy to monitor the accumulated fatigue damage in real-time, employing a joint input-state estimation algorithm. Measuring the operational vibrations at well-chosen locations enables the estimation of strain responses at unmeasured locations. The estimation algorithm is applied to a wind turbine on a lattice support structure, for which the response estimates of the lattice members are based on measurements on the turbine tower only. This restriction follows from the difficulty to reliably and robustly measure at locations on the lattice structure. Artificial measurement data is generated with a full-order finite element model, while the strains are estimated with an erroneous reduced-order design model, after inclusion of measurement noise. The strain estimates show that the main frequency content can be captured relatively accurately, except for a small bias and some high frequency disturbance, corresponding to a weakly observable higher mode. This second aspect shows the importance of a trade-off between the accuracy of the reduced-order finite element model and the ill-conditioning of the observability matrix.
\end{abstract}

Keywords: Offshore wind, response estimation, operational vibrations, lattice structure, sensor network

\section{Introduction}

For offshore wind turbine support structures, fatigue is a main design driver [1]. Fatigue-based design requires an adequate prediction of the environmental conditions combined with an accurate description of the structural properties, valid for the design life-time of, for example, 20 years. Estimates of the accumulated fatigue damage are, however, characterized by a large degree of uncertainty. This uncertainty stems from a) the loading specifications and b) the numerical models used to predict the response.

Given the random nature of both aerodynamic and hydrodynamic conditions, and the dependency on the structural response of the corresponding forces, a large number of scenarios needs to be evaluated, each of which relating the operational state of the turbine to the state of the environment. The aerodynamics within an offshore wind farm are not yet fully understood and moreover, the calculation of the loads on a rotating blade attached to a vibrating tower is generally based on inaccurate aerofoil data [2]. Considering hydrodynamics, the choice of an appropriate wave climate and a corresponding wave load calculation procedure is not straightforward [3]. Additional aspects, such as scour development, marine growth and secondary steel complicate the hydrodynamic analysis even further $[4,5]$. Since the aerodynamic and hydrodynamic actions on an offshore wind turbine are coupled through the structural response, the evaluation of the environmental conditions cannot be separated [6]. Similar difficulties are encountered when regarding the dynamic action due to sea ice [7].

Vorpahl et al. [8] conducted an extensive comparative study for a large number of relevant design calculation codes. Already for the estimation of the first natural frequency of a monopile-founded turbine, considerable scatter was observed. Furthermore, measurements of natural frequencies of installed offshore wind turbines showed higher values than designed for [9]. Unsufficient understanding of the interaction between the structure and the soil in terms of damping [10] and stiffness [9] could be an important source of this deviation. Zaaijer [11] considered the uncertainty regarding the foundation design, which potentially leads to large discrepancies between modelled and observed dynamic behaviour.

The uncertainty in both loading and modelling necessitates the use of safety factors, which most likely lead to overly conservative designs or underestimated service lives. Real-time monitoring of the strains in existing wind turbine support structures would provide us with knowledge of the actual fatigue damage accumulation, enabling an estimation of the remaining service life-time. One approach could be to continually monitor the strain at a 
number of critical locations. Knowing these locations beforehand, however, could be problematic - consider for instance a lattice structure. Moreover, the failure of a single sensor could frustrate the monitoring process considerably. A need thus exists for a robust integrated fatigue monitoring strategy capable of decreasing the current uncertainty regarding damage development in support structures offshore.

With the exception of [12], very little has been done with respect to integrated structural health monitoring of offshore wind turbines. Their focus, however, was on damage detection, sensor-fault detection and load identification. In this contribution, the strain response in a lattice support structure - allowing for the estimation of the accumulated fatigue damage - is estimated on the basis of operational vibrations using a joint input-state estimation algorithm proposed by Lourens et al. [13], in which it is assumed that no prior knowledge of the dynamic characteristics of the input forces is available. Particular attention is paid to the placement of the sensors, which should be within reach for maintenance. The choice for a lattice support structure follows from the expectation that in moderate water depths its application will be more common. Previous studies to the dynamics of lattice support structures can be found in [14] and [15].

To generate artificial measurement data, a reference finite element model, consisting of a simplified wind turbine on a lattice foundation, is constructed. The response data results from combined aerodynamic and hydrodynamic loading. After inclusion of measurement noise, the generated data and an erroneous design model are used to estimate the input forces, the states, and subsequently the strains required to predict fatigue. The erroneous model deviates significantly from the true finite element model, illustrating that despite a relatively weak model representation, the accumulated fatigue damage can be estimated accurately. Apart from the deliberate inclusion of modelling errors, Papadimitriou et al. [16] presented a similar, successful application of the joint input-state estimator for fatigue prediction.

\section{Method}

\section{$2.1 \quad$ Joint input-state estimation}

The joint input-state estimator used for the current analysis was presented by Lourens et al. [13]. The algorithm allows for the estimation of states, in terms of displacements and velocities, and the input forces on the basis of a limited number of measurement signals, displacements and accelerations, given that the location of the input forces is known. The starting point of the estimation algorithm is the modally reduced formulation of the system under consideration:

$$
\ddot{\mathbf{q}}(t)+\Gamma \dot{\mathbf{q}}(t)+\mathbf{\Omega}^{2} \mathbf{q}(t)+\boldsymbol{\Phi}^{\mathrm{T}} \mathbf{S}_{\mathbf{p}} \mathbf{p}(t)
$$

Here, $\mathbf{q}(t) \in \mathbb{R}^{n_{m}}$ represents the vector of generalized coordinates and $\mathbf{p}(t) \in \mathbb{R}^{n_{p}}$ the input force vector, with $n_{m}$ the number of modes and $n_{p}$ the number of input forces. The matrix $\Gamma \in \mathbb{R}^{n_{m} \times n_{m}}$ is the modal damping matrix and $\boldsymbol{\Omega} \in \mathbb{R}^{n_{m} \times n_{m}}$ a diagonal matrix, containing the natural frequencies related to the $n_{m}$ modes on its diagonal. The corresponding mass normalized mode shapes are collected in the matrix $\boldsymbol{\Phi} \in \mathbb{R}^{n_{d o f} \times n_{m}}$, with $n_{d o f}$ the number of degrees of freedom of the unreduced space-discretized model, and the mode vectors $\boldsymbol{\varphi}_{j}$, for $j=1, \ldots, n_{m}$ as its columns. The force selection matrix $\mathbf{S}_{\mathbf{p}} \in \mathbb{R}^{n_{d o f} \times n_{p}}$ specifies the force locations. A dot indicates a derivative with respect to time and the superscript $\mathrm{T}$ implies a transpose.

The measured quantities are combined in the output vector $\mathbf{d}(t) \in \mathbb{R}^{n_{d}}$, with $n_{d}$ the number of measured locations:

$$
\mathbf{d}(t)=\mathbf{S}_{\mathbf{a}} \boldsymbol{\Phi} \ddot{\mathbf{q}}(t)+\mathbf{S}_{\mathbf{v}} \boldsymbol{\Phi} \dot{\mathbf{q}}(t)+\mathbf{S}_{\mathbf{d}} \mathbf{\Phi q}(t),
$$

The selection matrices $\mathbf{S}_{\mathbf{a}}, \mathbf{S}_{\mathbf{v}}$ and $\mathbf{S}_{\mathbf{d}} \in \mathbb{R}^{n_{d o f} \times n_{d}}$ specify the locations of the acceleration, velocity and displacement/strain measurements, respectively. After adopting the state-space formulation for both Eq. (1) and (2), where $\mathbf{x}(t)=\left[\begin{array}{ll}\mathbf{q}(t) & \dot{\mathbf{q}}(t)\end{array}\right]^{\mathrm{T}}$, and discretizing the continuous-time components, the system can be rewritten in terms of the following discrete-time combined deterministic-stochastic state-space model [13]:

$$
\mathbf{x}_{k+1}=\mathbf{A} \mathbf{x}_{k}+\mathbf{B} \mathbf{p}_{k}+\mathbf{w}_{k}
$$




$$
\mathbf{d}_{k}=\mathbf{G} \mathbf{x}_{k}+\mathbf{J} \mathbf{p}_{k}+\mathbf{v}_{k}
$$

with the discretizations $\mathbf{x}_{k}=\mathbf{x}(k \Delta t), \mathbf{p}_{k}=\mathbf{p}(k \Delta t)$ and $\mathbf{d}_{k}=\mathbf{d}(k \Delta t)$, for $k=1, \ldots, N$, where $\Delta t$ is the sampling time step and $N$ is the number of samples. The matrices $\mathbf{A} \in \mathbb{R}^{2 n_{m} \times 2 n_{m}}$ and $\mathbf{B} \in \mathbb{R}^{2 n_{m} \times n_{p}}$ represent the discretized system matrices, that can be related to their time-continuous counterparts $\mathbf{A}_{\mathbf{c}}$ and $\mathbf{B}_{\mathbf{c}}$ in the following manner:

$$
\begin{gathered}
\mathbf{A}=\mathrm{e}^{\mathbf{A}_{\mathbf{c}} \Delta t} \\
\mathbf{B}=[\mathbf{A}-\mathbf{I}] \mathbf{A}_{\mathbf{c}}^{-\mathbf{1}} \mathbf{B}_{\mathbf{c}}
\end{gathered}
$$

with the identity matrix $\mathbf{I} \in \mathbb{R}^{2 n_{m} \times 2 n_{m}}$. The output influence matrix $\mathbf{G} \in \mathbb{R}^{n_{d} \times 2 n_{m}}$ and the direct transmission matrix $\mathbf{J} \in \mathbb{R}^{n_{d} \times n_{p}}$ are defined as

$$
\begin{aligned}
& \mathbf{G}=\left[\mathbf{S}_{\mathrm{d}} \boldsymbol{\Phi}-\mathbf{S}_{\mathrm{a}} \boldsymbol{\Phi} \boldsymbol{\Omega}^{2} \quad \mathbf{S}_{\mathbf{v}} \boldsymbol{\Phi}-\mathbf{S}_{\mathrm{a}} \boldsymbol{\Phi} \boldsymbol{\Gamma}\right] \\
& \mathbf{J}=\left[\mathbf{S}_{\mathbf{a}} \boldsymbol{\Phi} \boldsymbol{\Phi}^{\mathrm{T}} \mathbf{S}_{\mathbf{p}}\right]
\end{aligned}
$$

Process and measurement noise, resulting, respectively, from unmodelled inputs or modelling errors and sensor inaccuracies, are represented by the stochastic components $\mathbf{w}_{k} \in \mathbb{R}^{2 n_{m}}$ and $\mathbf{v}_{k} \in \mathbb{R}^{n_{d}}$. These noise processes are assumed to be stationary, zero-mean and white. Furthermore, the noise processes $\mathbf{w}_{k}$ and $\mathbf{v}_{k}$ are assumed to be uncorrelated. The joint input-state estimation algorithm requires the covariance matrices for the separate noise processes, represented by $\mathbf{Q}=\mathrm{E}\left\{\mathbf{w}_{k} \mathbf{w}_{l}^{\mathrm{T}}\right\} \in \mathbb{R}^{2 n_{m} \times 2 n_{m}}$ and $\mathbf{R}=\mathrm{E}\left\{\mathbf{v}_{k} \mathbf{v}_{l}^{\mathrm{T}}\right\} \in \mathbb{R}^{n_{d} \times n_{d}}$, for $k, l=1, \ldots, N$, to be known. Additionally, the algorithm requires an initial unbiased state estimate $\hat{\mathbf{x}}_{0}$, where the hat indicates an estimated quantity, and its error covariance matrix $\mathbf{P}_{0} \in \mathbb{R}^{2 n_{m} \times 2 n_{m}}$ to be available.

The estimation algorithm represents an extension of the filter presented by Gillijns and De Moor [17], developed for linear systems with direct transmission. This filter was proved to be optimally in estimated states and inputs in a minimum-variance unbiased sense. The extension stems from the numerical instabilities which arise when $n_{d}$ exceeds $n_{m}$. This instability issue can be circumvented by limiting the order of the potentially rank-deficient matrix multiplications to the number of modes accounted for [13].

A contribution by Maes et al. [18] generalizes the joint input-state estimator by allowing for correlation between the processes $\mathbf{w}_{k}$ and $\mathbf{v}_{k}$ via the correlation matrix $\mathbf{S} \in \mathbb{R}^{2 n_{m} \times n_{d}}$ :

$$
\mathbf{S}=\mathrm{E}\left\{\mathbf{w}_{k} \mathbf{v}_{l}^{\mathrm{T}}\right\}
$$

Under the assumption of white noise stochastic input processes, the matrix entries of $\mathbf{S}$ can be estimated from operational data. Since the stochastic input processes in the specific case under consideration, an offshore wind turbine, are not close to being white, this generalization is not accounted for.

\subsection{Response estimation}

With the estimated force time histories $\hat{\mathbf{p}}_{k} \in \mathbb{R}^{n_{p}}$ and state sequences $\hat{\mathbf{x}}_{k} \in \mathbb{R}^{2 n_{m}}$, response predictions $\hat{\mathbf{d}}_{k} \in \mathbb{R}^{n_{r}}$ at $n_{r}$ unmeasured locations can be constructed. Hereto, the observation equation (Eq. (4)) is employed [16]:

$$
\hat{\mathbf{d}}_{k}=\mathbf{G} \hat{\mathbf{x}}_{k}+\mathbf{J} \hat{\mathbf{p}}_{k}
$$

It should be noted that the output influence matrix $\mathbf{G}$ and the direct transmission matrix $\mathbf{J}$ are now constructed to correspond to the response prediction, for which the selection matrices $\mathbf{S}_{\mathbf{a}}, \mathbf{S}_{\mathbf{v}}$ and $\mathbf{S}_{\mathbf{d}}$ specify the locations, as well as the type of response to be predicted.

The response estimation does not require the locations of the input forces to be accurately known. If the location of the input forces is unknown, the joint input-state estimator can be applied to estimate equivalent forces at arbitrarily chosen locations, causing the same measured response. Furthermore, if an erroneous model is applied 
for the response estimation, the force estimations could potentially serve to reduce the effects of the modelling errors on the response predictions, as will be shown in Section 3.2

\subsection{Wind turbine model}

The analysis is based on a lattice structure, as described by De Vries et al. [15], supporting a 5 MW reference turbine, for which the main characteristics are presented by Jonkman et al. [19]. Fig. 1 illustrates the geometry of the combined turbine and support structure, including the main geometric and material characteristics. The combined turbine and lattice structure is modelled in 2D by means of the finite element method. Euler-Bernoulli beam elements, possessing six degrees of freedom, are employed to represent the steel members and the turbine tower. The rotor-nacelle assembly is reduced to a lumped mass and at the jacket base the structure is connected rigidly fixed to the soil. Compression due to the self-weight of the structure, which reduces the effective stiffness, is not accounted for. By varying the stiffness characteristics of the concrete transition piece, the model is updated such, that the first two natural frequencies show good agreement with those presented in [15].

As a basis for the joint input-state estimation, a modal representation of the wind turbine model is required, see Eq. (1). Fig. 2 presents the first ten mode shapes of the modelled turbine structure. The natural frequencies of the first ten modes, including a brief description of the mode, are presented in Table 1. Structural damping is accounted for in terms of classical modal damping, i.e. a diagonal damping matrix is adopted. For each mode, a damping value of $1.0 \%$ of the critical damping is assumed.

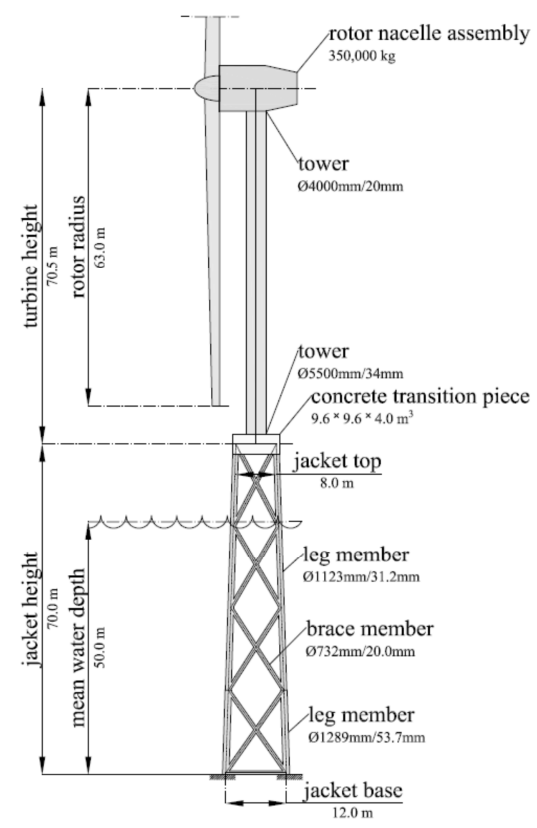
support structure

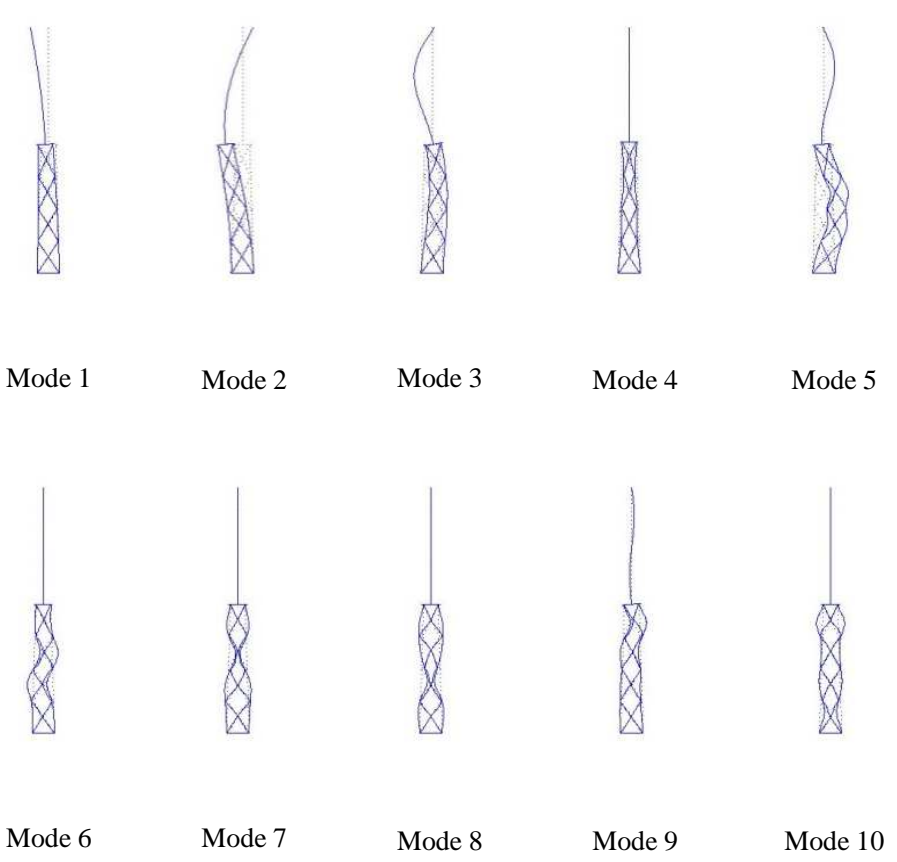

Fig. 2 Results of the finite element modal analysis for the first ten modes

\begin{tabular}{lll}
\hline No. & Natural frequency $[\mathrm{Hz}]$ & Description \\
\hline 1 & 0.364 & First global lateral mode \\
2 & 1.07 & Second global lateral mode \\
3 & 4.89 & Third global lateral mode \\
4 & 6.20 & First global vertical mode \\
5 & 6.77 & Fourth global lateral mode \\
6 & 10.4 & Lateral jacket mode (second and third frame from top - in-phase \\
7 & 10.4 & Local lateral jacket mode (second frame from top - anti-phase) \\
8 & 10.9 & Local lateral jacket mode (third frame from top - anti-phase) \\
9 & 12.6 & Local lateral jacket mode (first frame from top - in-phase) \\
10 & 13.8 & Local lateral jacket mode (first and fourth frame from top - anti-phase) \\
\hline
\end{tabular}

Table 1 Natural frequencies corresponding to the first ten modes, as derived from the finite element modal analysis 


\subsection{Stochastic wind force}

The time-dependent wind force is determined on the basis of the actuator disc concept, elaborated on in [20], where a 1D free field turbulence is simulated on the basis of the spectral properties of a Kaimal power density spectrum $S_{u u}$ as a function of frequency $f$ :

$$
S_{u u}(f)=\sigma_{u}^{2} \frac{4 L_{1 u} / \bar{U}}{\left(1+6 f L_{1 u} / \bar{U}\right)^{5 / 3}}, \text { for } f>0 \mathrm{~Hz}
$$

$\bar{U}$ represents the mean wind velocity, $L_{1 u}$ is a turbulence length scale and $\sigma_{u}$ is the standard deviation of the turbulence velocity. To generate a wind force signal, an optimally functioning turbine is assumed, implying that the turbine operates at the Lanchester-Betz limit. Furthermore, it is assumed that the induced velocity through the rotor disc follows the instantaneous turbulent wind velocity. This assumption implies that the entire wake changes instantaneously, such that equilibrium in the wake is maintained at all times.

Fig. 3 shows the Kaimal spectrum adopted for the generation of the wind force signal. The spectrum reveals a main energy contribution from the frequencies below $1 \mathrm{~Hz}$. Assuming a random phase distribution, and a cut-off frequency of $3 \mathrm{~Hz}$, a wind force signal of $546 \mathrm{~s}$ is generated, in accordance with [21]. Fig. 4(a) depicts a $100 \mathrm{~s}$ window of this time signal. Since the finite element model does not include a detailed rotor representation, the total wind force is assumed to act concentratedly at the rotor nacelle assembly at the tower top, as can be seen in Fig. 4(b). It should be noted that, despite the turbulence frequency cut-off, the wind force signal contains higher frequency contributions. This results from the nonlinear dependency of the wind force on the turbulent wind velocity.

The apparent damping, resulting from a rotating rotor, is assumed at $4.0 \%$ of the critical damping for the first structural mode. For higher modes, the contribution of this aerodynamic damping is scaled on the basis of the modal deflection at the tower top.

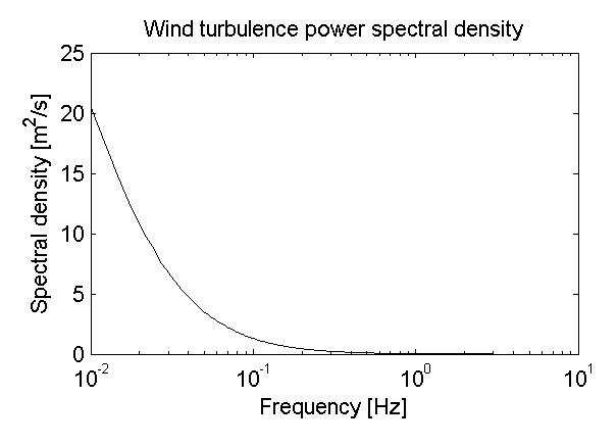

Fig. 3 Kaimal spectrum derived for mean wind velocity of $10 \mathrm{~m} / \mathrm{s}$, a turbulence intensity of $10 \%$ and a turbulence length scale of $150 \mathrm{~m}$

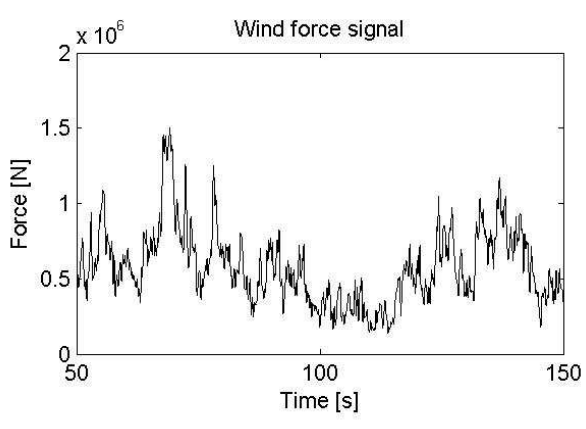

(a)

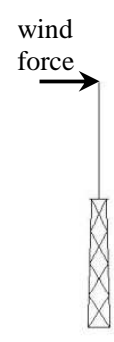

(b)

Fig. 4 (a) Turbulent-wind force signal for NREL5 offshore wind turbine, and (b) positioning of the wind force on the FE model

\subsection{Stochastic wave force}

The Morison equation is commonly adopted to estimate hydrodynamic actions on slender vertical members. The equations derive the total hydrodynamic force by superposing an inertia and a drag force contribution, depending on the wave particle acceleration and velocity, respectively. A procedure for defining a hydrodynamic force on the basis of the Morison equation, using the linear wave theory for defining the wave particle actions, is elaborated on in [22]. The original Morison equation was derived for vertically-oriented cylindrical piles and for very small pile diameter to wave length ratios. To determine the wave forces on the current lattice structure, some engineering adjustments have to be implemented. As an equivalent structural diameter, the combined width of the members exposed to the hydrodynamic action is taken. Equally, an equivalent cross-sectional area results from the sum from the different lattice elements. For the hydrodynamic inertia and drag coefficients, common values of 2.0 and 0.7 , respectively, are adopted.

The wave particle actions for a specific sea state can be derived from a relevant wave elevation spectrum. In this particular case, use is made of a Pierson-Moskovitz spectrum, depending on the mean wind velocity $\bar{U}$, see Fig. 5: 


$$
S_{h h}(f)=\alpha \frac{g^{2}}{(2 \pi f)^{5}} \mathrm{e}^{-\beta\left(\frac{g}{2 \pi f \bar{U}}\right)^{4}}
$$

where $\alpha=0.0081$ and $\beta=0.74$ are coefficients and $g$ is the gravitational acceleration. Compared to the wind turbulence spectrum of Fig. 3, the wave elevation spectrum contains its energy at somewhat higher frequencies. The peak energy is much smaller. On the basis of the wave elevation distribution, the associated wave kinematics can be determined.

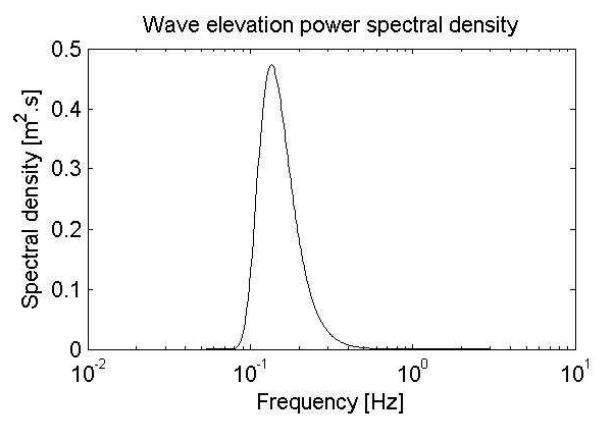

Fig. 5 Pierson-Moskowitz spectrum derived for a mean wind velocity of $10 \mathrm{~m} / \mathrm{s}$
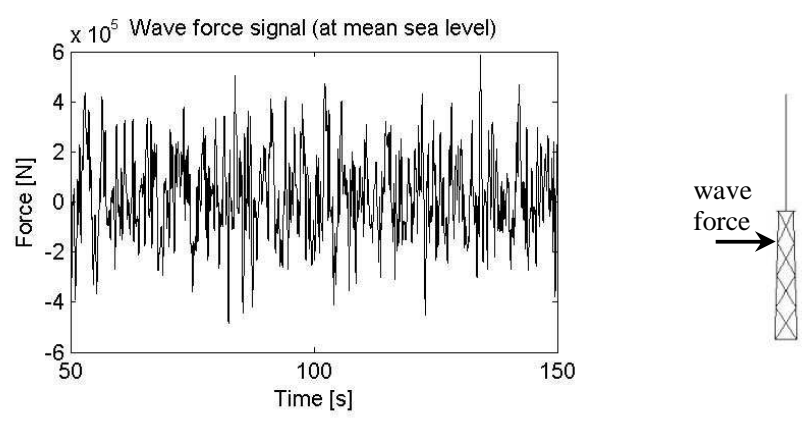

(a)

(b)

Fig. 6 (a) Wave force signal for on lattice structure, and (b) positioning of the wave force on the finite element model

The magnitude of the distributed wave force is calculated at mean sea level. This force is assumed to act within a wave impact zone of $\pm 5.0 \mathrm{~m}$ with respect to this level. After integration over this wave impact zone, a concentrated wave force signal at mean sea level is derived. In deriving this force signal, of which a $100 \mathrm{~s}$ window is presented in Fig. 6(a), a $1.0 \mathrm{~m} / \mathrm{s}$ current is added to the wave particle velocity. In correspondence to the wind force generation, the signal has a length of $546 \mathrm{~s}$, while a cut-off frequency of $3 \mathrm{~Hz}$ has been adopted. The force location is chosen at the K-joint, connecting the first and second jacket frame, see Fig. 6(b). Since the Morison equation relates the drag force nonlinearly to the wave particle velocity, the wave force contains frequency content above the cut-off frequency of the wave elevation energy spectrum.

The added damping resulting from the hydrodynamic action is neglected. This hydrodynamic damping would result from the response velocity of the jacket members and its contribution can be assumed to be small.

\subsection{Sensor network}

Given the hostile environmental offshore conditions, the measurement of the structural motion requires a robust network of sensors. This robustness is first pronounced in the number of required sensors: if only a limited number of sensors is needed, the costs to build in sufficient redundancy remain low. Second, the positioning of the sensors affects the robustness of the system. This implies that no sensors should be placed under the water level, because these locations are not easily reached when maintenance is necessary. Moreover, to prevent sensors from early failure, sensors within the wave splash zone should be avoided. Therefore, the estimation of the dynamic response will be based on a network consisting of sensors attached to the turbine tower only.

The design of a sensor network for optimal estimation of inputs and states is considered by Maes et al. [23]. In this contribution, the criteria for the invertibility of a system, and therefore the possible application of the joint input-state estimator, are derived in terms of stability and identifiability. For the joint input-state estimator to be stable, two requirements need to be fulfilled. First, the number of acceleration and/or velocity sensors, $n_{d ; a}$ and $n_{d ; v}$, respectively, needs to be equal to or larger than the number of input forces:

$$
n_{d ; a}+n_{d ; v} \geq n_{p}
$$

Second, the number of displacement/strain sensors $n_{d ; d}$ needs to be equal to or larger than the number of input forces:

$$
n_{d ; d} \geq n_{p}
$$


In this particular case, the separate estimation of the wind and wave force requires a network consisting of two acceleration and/or velocity sensors and two displacement/strain sensors.

Identifiability relates to the controllability of the input forces, the observability of the states and the direct invertibility of the measurement outputs towards states and input. The controllability of the input forces can by assessed by determining the rank of the controllability matrix $\mathbf{C} \in \mathbb{R}^{2 n_{m} \times\left(n_{p} \times 2 n_{m}\right)}$ :

$$
\mathbf{C} \equiv\left[\begin{array}{llll}
\mathbf{B} & \mathbf{A B} & \ldots & \mathbf{A}^{2 n_{m}-1} \mathbf{B}
\end{array}\right]
$$

If $\mathbf{C}$ is of full rank, i.e. $\operatorname{rank}(\mathbf{C})=2 n_{m}$, the system is controllable, implying that the assumed forces are positioned such, that all modes can contribute to the response. Alternatively, the controllability can be assessed by determining the rank of the modal projections of the force selection matrix $\mathbf{S}_{\mathbf{p}} \boldsymbol{\varphi}_{j}$, for which it should apply that $\operatorname{rank}\left(\mathbf{S}_{\mathbf{p}} \boldsymbol{\varphi}_{j}\right)=1$ for $j=1, \ldots, n_{m}$.

In a similar manner, the observability of the system can be assessed by determining the rank of the observability matrix $\mathbf{H} \in \mathbb{R}^{\left(2 n_{m} \times n_{d}\right) \times 2 n_{m}}$ :

$$
\mathbf{H} \equiv\left[\begin{array}{c}
\mathbf{G} \\
\mathbf{G A} \\
\vdots \\
\mathbf{G A}^{2 n_{m}-1}
\end{array}\right]
$$

If $\mathbf{H}$ is of full rank, i.e. $\operatorname{rank}(\mathbf{H})=2 n_{m}$, the system is observable, implying that the sensors are positioned such, that all states can be identified uniquely, irrespective of the initial conditions. Alternatively, the observability can be assessed by determining the rank of the modal projections of the sensor selection matrix $\mathbf{S}_{\mathbf{d}} \boldsymbol{\varphi}_{j}$, for which it should apply that $\operatorname{rank}\left(\mathbf{S}_{\mathbf{d}} \boldsymbol{\varphi}_{j}\right)=1$ for $j=1, \ldots, n_{m}$.

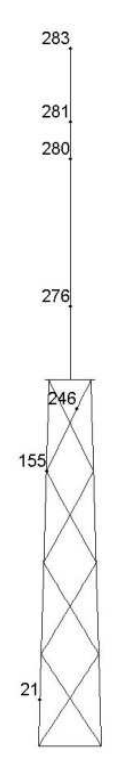

Fig. 7 Selected nodes for force positioning, sensor placement and response estimations
Direct invertibility requires the number of structural modes accounted for with the reduced-order model to be equal to or larger than the number of forces to be identified, i.e. $n_{m} \geq n_{p}$. The implication of direct invertibility is that input forces can be estimated from the system output, without delay in time.

Fig. 7 presents the finite element model of the structure, where a number of nodes is specifically indicated. The known locations of the wind and wave force, respectively, are node 283 and node 155 . The four sensor locations are node 276, 280, 281 and 283. The remaining nodes, 21 and 246, will serve as response estimation locations in Section 3.1 and 3.2. These locations are chosen to illustrate the difference in quality of response estimations over the height of the lattice structure. Particularly for node 21, the estimate is expected to be inaccurate, due to the large distance from the sensor locations [24].

The modal projections $\mathbf{S}_{\mathbf{p}} \boldsymbol{\varphi}_{j}$ for the first ten modes are depicted in Fig. 8. It can be clearly seen that the wind force location projects mainly on the first mode. Mode 2 and 3 could serve the wind force identification too. The modal projection of the wave force location is highest for the higher modes: mode 5, 8, 9 and 10 . Given the frequency content of the wave force, however, not much excitation of these higher modes is to be expected.

Fig. 9 shows the modal projections $\mathbf{S}_{\mathbf{d}} \boldsymbol{\varphi}_{j}$ of the four sensor locations for the first ten structural modes. The sensors only measure lateral motions. Based on this figure, it can be concluded that positioning four sensors at the chosen tower locations enables the identification of states related to mode $1,2,3,5$ and 9 . It is clear that for some modes the modal projection is very small. Mode 
4 , for instance, represents a vertical global mode, for which the states are difficult to capture with sensors that only function laterally. Mode 6, 7, 8 and 10 represent local jacket modes, with very small tower amplitudes.

Combining Fig. 8 and Fig. 9, it can be concluded that the network consisting of sensors on the tower only will not allow for the dynamic response estimation resulting from higher mode excitation. The frequency content of both turbulence and wave elevation is cut off at $3 \mathrm{~Hz}$, implying that the frequency content of the wind and wave force above $3 \mathrm{~Hz}$ will be limited - despite the nonlinear force formulation. Still, the inclusion of mode 4, 7, 8 and 10 in the estimation analysis results in a numerically rank-deficient observability matrix, implying that the system is ill-conditioned. To a lesser extent, this also applies for mode 6 . To prevent the system from being illconditioned, the modes $4,7,8$ and 10 are excluded from further analysis, leaving a reduced-order model consisting of six modes. A convergence analysis with respect to the full-order model has shown that the reducedorder model enables the generation of accurate measurement signals. If mode 6 is excluded from the analysis too, the ill-conditioning of the observability matrix would be reduced. This further reduction of the modal basis would, however, have too large an effect on the accuracy of the model.

The adopted sensor network consists of accelerometers at node 281 and 283 and strain gauges at node 276 and 280. The reasoning behind is that the accelerations are expected to be largest near the tower top. Strains, on the other hand, are more pronounced in the lower section of the tower.

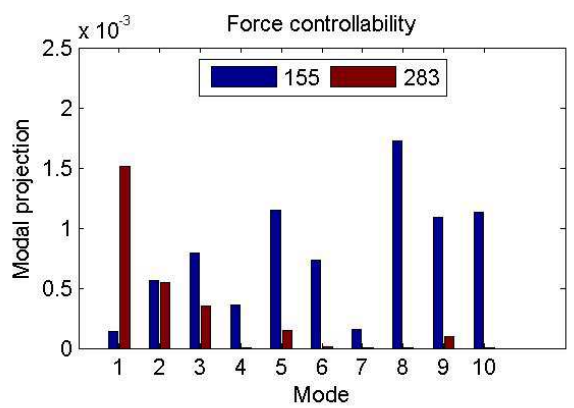

Fig. 8 Modal projections to the wave force location (node 155) and the wind force location (node 283)

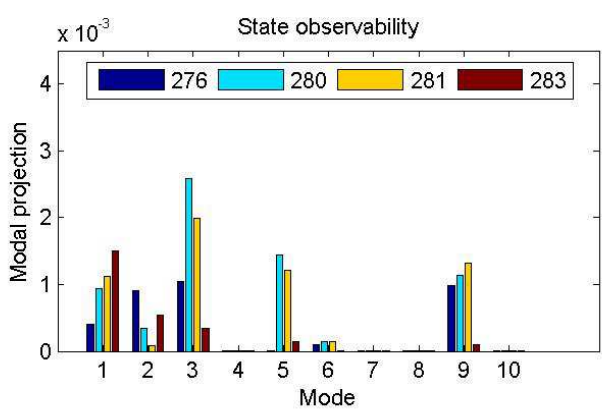

Fig. 9 Modal projections to the sensor locations, node 276 , node 280 , node 281 and node 283

\section{Results}

\subsection{Response estimation in the absence of modelling errors}

First, the response prediction is tested for the robustness to the inclusion of measurement noise only. Application of the force time signals to the finite element model results in simulated measurement data at the chosen sensor locations. To the measurement signals $\mathbf{d}_{k}$, consisting of $N$ time samples each, some Gaussian white noise is added, resulting in the polluted output vector $\tilde{\mathbf{d}}_{k} \in \mathbb{R}^{n_{d}}$ per time step $k$. Given the stationary random nature of the measurement signals, the noise is chosen to be related to the standard deviation of the separate measurements, $\sigma_{\mathrm{d}} \in \mathbb{R}^{n_{d} \times n_{d}}$ :

$$
\tilde{\mathbf{d}}_{k}=\mathbf{d}_{k}+\gamma \boldsymbol{\sigma}_{\mathbf{d}} \mathbf{r}_{k}
$$

where $\gamma$ represents the noise level and $\boldsymbol{\sigma}_{\mathrm{d}}$ is a diagonal matrix with the standard deviations of the time signals as its diagonal entries. $\mathbf{r}_{k} \in \mathbb{R}^{n_{d}}$ is a vector composed of random values taken from a normal distribution with zero mean and a standard deviation of one.

For $\gamma$ a value of 0.05 is applied, corresponding to 5\% measurement noise. Eq. (17) allows for the exact calculation of the entries of the measurement covariance matrix $\mathbf{R}$ :

$$
\mathbf{R}=\gamma \boldsymbol{\sigma}_{\mathbf{d}}^{2}
$$

The initial states are assumed to be zero. Since no process noise is present, the entries of the error covariance matrices $\mathbf{P}_{0}$ and $\mathbf{Q}$ are chosen very small, namely $1 \cdot 10^{-20}$. 
The prediction of the response in the lattice structure requires force and state estimations, obtained by means of the joint input-state estimator from the noise-contaminated measurement signals. These estimated forces and states, $\hat{\mathbf{p}}_{k}$ and $\hat{\mathbf{x}}_{k}$, respectively, are subsequently used to estimate the response as described in Section 2.2. Since the modal basis consists of six modes, a total of twelve states is estimated by the estimation algorithm. Given the main frequency content of the force signals - below $3 \mathrm{~Hz}$ - and the natural frequencies of the system, the estimation of the first and second modal states are most relevant. Fig. 10 presents the state estimation corresponding to these modes. The inclusion of the measurement noise still allows an accurate estimation of the first modal states. The estimation of the second modal states is shown to be obviously more noisy.

Using Eq. (10), the strain estimates are derived at node 21 and 246 (see Fig. 7) and presented in Fig. 11. These particular locations are chosen to illustrate to what extent the response of lattice members can be estimated by means of tower measurements only. Fig. 11 shows that the low-frequency strain response is captured relatively well, despite the noise-contamination of the measurement signals. Still, some high-frequency disturbance in the estimations can be observed. The disturbance is most pronounced at $10.4 \mathrm{~Hz}$, which corresponds to the sixth natural frequency of the structure, a mode that is weakly observed by the sensor network. The response estimation can be improved by excluding mode 6 from the modal basis. This, however, would also weaken the accuracy of the reduced-order finite element model - see Section 3.2 for a more detailed discussion.

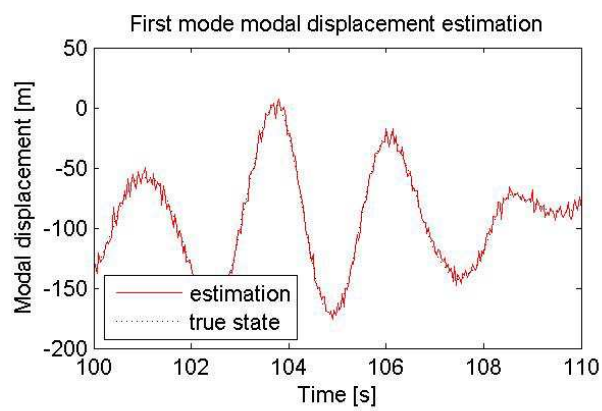

(a)

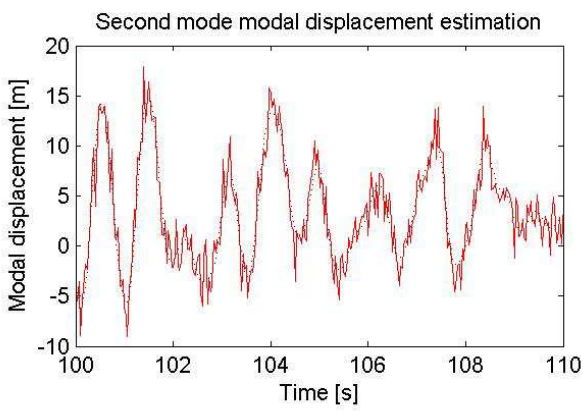

(c)

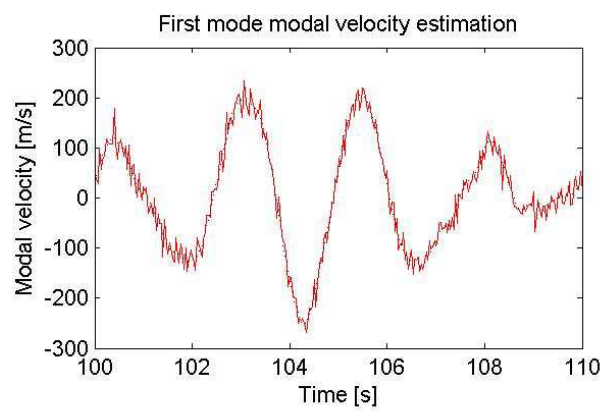

(b)

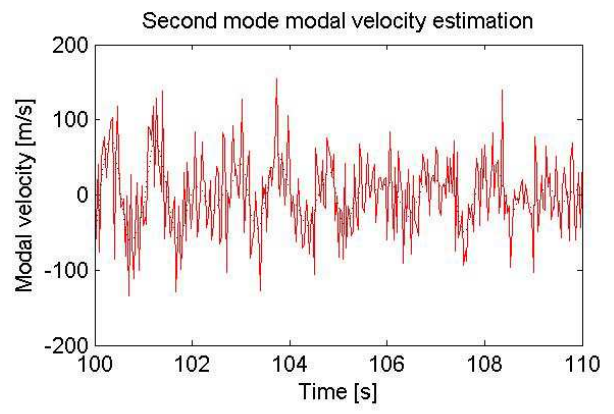

(d)

Fig. 10 State estimation for (a) the modal displacement of mode 1, (b) the modal velocity of mode 1, (c) the modal displacement of mode 2 and (d) the modal velocity of mode 2

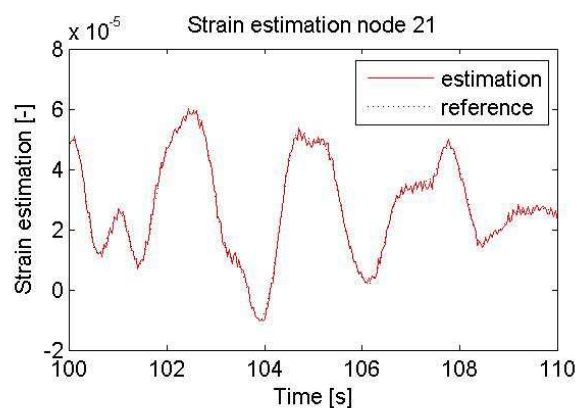

(a)

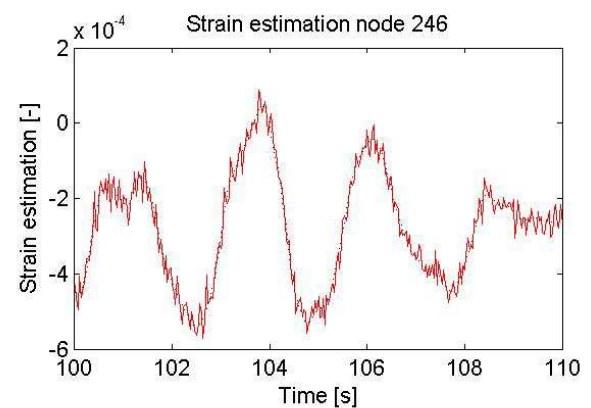

(b)

Fig. 11 Time signal representation of the strain response estimation at two lattice members: (a) node 21 and (b) node 246 


\subsection{Response estimation with an erroneous model}

A second response estimation is performed, this time with a design model that does not exactly represent the true structure. Process noise is deliberately introduced in the design model, by increasing the first and second natural frequency with $20 \%$. The first and second natural frequency of the design model are $0.437 \mathrm{~Hz}$ and $1.21 \mathrm{~Hz}$, instead of $0.364 \mathrm{~Hz}$ and $1.07 \mathrm{~Hz}$ of the model with which the data is generated. Again, the measurement data is polluted with $5 \%$ measurement noise.

The inclusion of process noise complicates the estimation of the true forces and states. Instead, the joint inputstate estimator enables the estimation of equivalent forces and states, which combined enable the response estimation at unmeasured structure locations. To lesser extent, this was already observed in the previous section, where only measurement noise was accounted for. To optimize the functioning of the estimation algorithm, the entries of the $\mathbf{Q}$ matrix are adjusted. The covariance of the process noise cannot be as easily estimated as the covariance of the measurement noise. By choosing the square roots of the process noise covariance entries of the same order of magnitude as a small percentage of the states, the covariance matrix entries can be made to correspond with what they represent [24].

When considering the frequency content of the estimated wind and wave forces, see Fig. 12 and Fig. 13, the force signals clearly compensate for the unmodelled dynamics. The corrective forces contain frequency peaks at the true natural frequencies The corresponding time signals illustrate the deviation of the estimated force from the true force. The effect of the measurement noise is mainly expressed as the high frequent disturbance of the wave force estimation. The compensation of the frequency content at the true natural frequencies enables a relatively accurate estimation of the first and second modal states, see Fig. 14. Despite a small bias, the estimated states show good correspondence with the true states.

The small bias in the state estimates can be recognized too in the estimated strain response at node 21 and 246. Fig. 15 presents short samples of the estimated time signals. This bias is a direct result of the invalidity of the zero-mean assumption used for the process noise $\mathbf{w}_{k}$. The magnitude of the bias, however, remains unaffected over the length of the time signals, implying that the bias does not corrupt possible fatigue damage estimates. Particularly at node 21, a noisy component in the response estimation is observed. To further asses this noisiness, the frequency content of the estimates is depicted in Fig. 16. It becomes clear that the sixth mode contributes excessively to the identified response. This result was already expected when the observability of the states was discussed (see Fig. 9), where it was expected that the inclusion of mode 6 increases the ill-conditioning of the observability matrix.

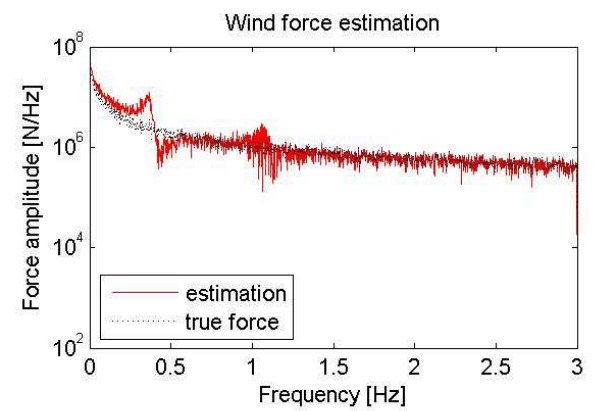

(a)

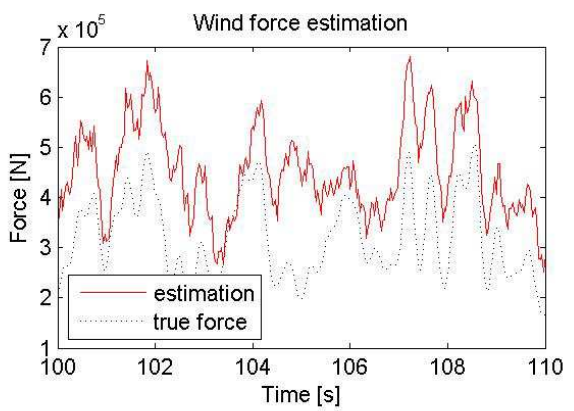

(b)

Fig. 12 Wind force estimation with erroneous design model, based on measurements with and without measurements noise, presented in (a) the frequency-domain and (b) the time-domain 


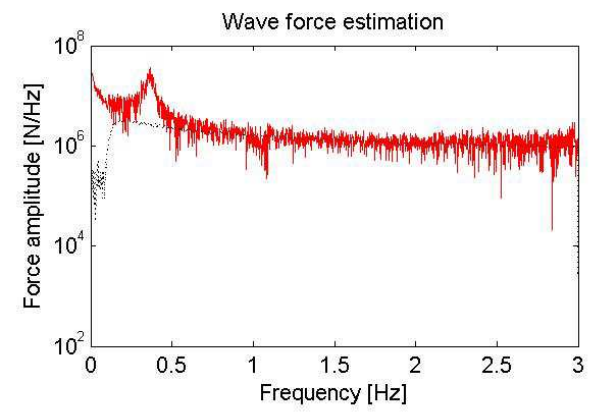

(a)

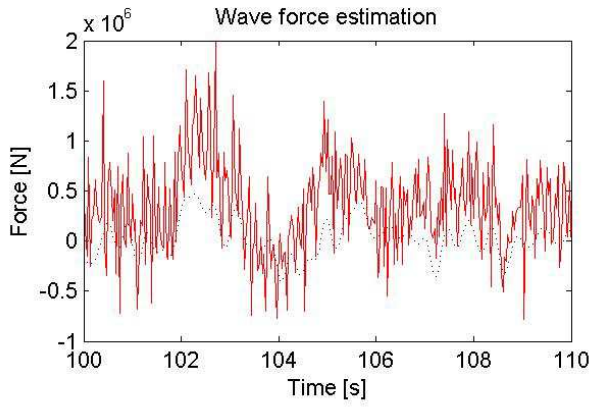

(b)

Fig. 13 Wave force estimation with erroneous design model, based on measurements with measurements noise, presented in (a) the frequency-domain and (b) the time-domain

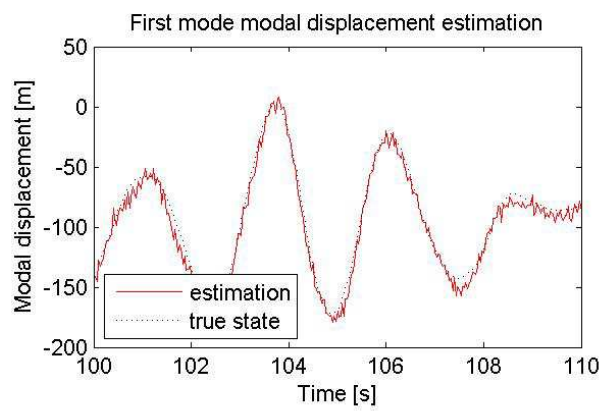

(a)

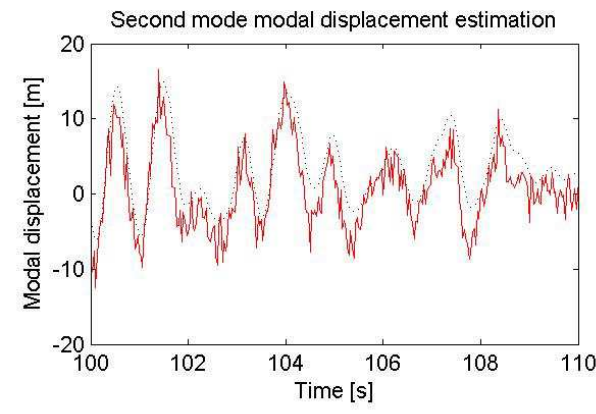

(c)

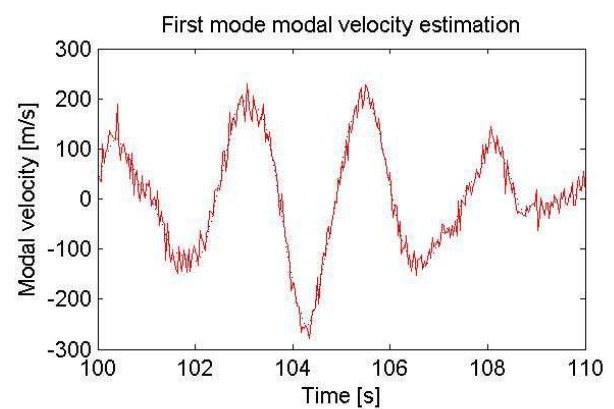

(b)

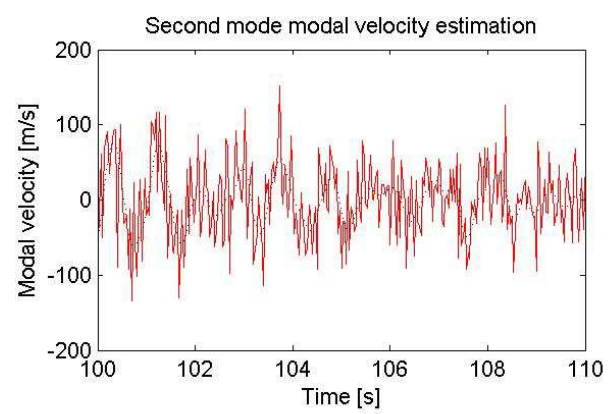

(d)

Fig. 14 State estimation with erroneous design model for (a) the modal displacement of mode 1, (b) the modal velocity of mode $1,(\mathrm{c})$ the modal displacement of mode 2 and (d) the modal velocity of mode 2

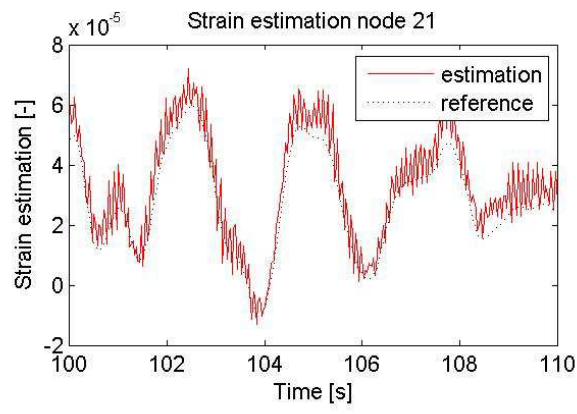

(a)

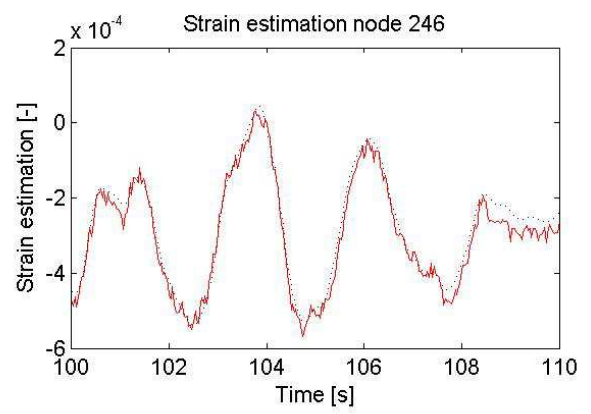

(b)

Fig. 15 Time signal representation of the strain response estimation with erroneous design model at two lattice members: (a) node 21 and (b) node 246 


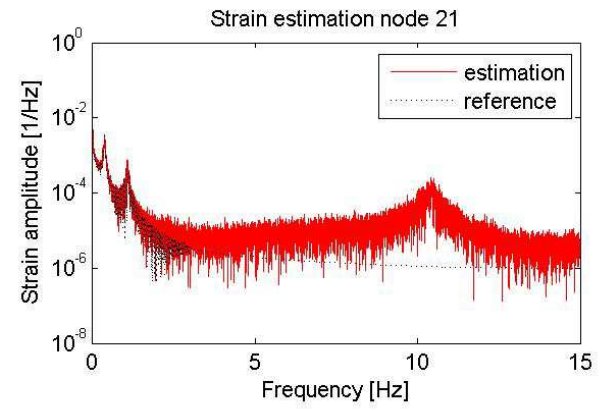

(a)

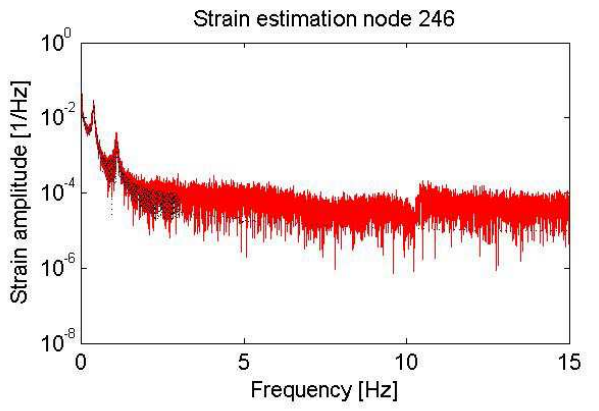

(b)

Fig. 16 Frequency domain representation of the strain response estimation with erroneous design model at two lattice members: (a) node 21 and (b) node 246

\section{$4 \quad$ Discussion and conclusions}

Recognizing that the design for fatigue for offshore wind turbines is characterized by uncertainty, while being a main design driver for this type of structures, this study presents a strategy for the real-time monitoring of the accumulated fatigue damage, employing a joint input-state estimation algorithm. In particular, a wind turbine on a lattice support structure is considered, for which the response estimates of the lattice members are based on measurements at the turbine tower only. This restriction follows from the difficulty to reliably and robustly measure at location on the lattice structure.

The study is based on a 2D finite element model representing the true offshore wind turbine. Measurement signals are generated from the response to known wind and wave forces, which are defined from conventional load models. First, inputs and states are estimated from measurements with a noise level of 5\% with a finite element model in the absence of modelling errors. From this, the strain response at chosen locations on the lattice structure is estimated, which could eventually serve the estimation of the accumulated fatigue damage. Subsequently, a design finite element model is constructed by adjusting the main natural frequencies. This design model is applied to estimate the strain response in the lattice structure on the basis of the true response measurements.

The response estimates show that the main frequency content can be captured relatively accurately. Also the low frequency response amplitudes correspond well to the real amplitudes. The estimations with the design model, with a $20 \%$ error on the first and second natural frequency, do show a small bias with respect to the real response, resulting from the invalidity of the zero-mean assumption used for the process noise. This bias, however, will not harm the quality of the accumulated fatigue damage estimation, since for this only the magnitude of the strain cycles is of interest. Nevertheless, the strain response estimates do contain some high frequency disturbance in which the sixth natural frequency of the system is most pronounced. The occurrence of this disturbance can be related to the ill-conditioning of the observability matrix.

The analysis illustrated the trade-off between the accuracy of the reduced-order finite element model and the illconditioning of the observability matrix. The modal basis of the finite element model should account for a sufficient number of modes to describe the dynamic response sufficiently accurate. Certain modes, however, can be hardly observable, implying ill-conditioning of the observability matrix. In this particular case, the observability of mode 6 is small, resulting in the noisy disturbance of the estimated response. Excluding mode 6 from the modal basis would decrease the ill-conditioning of the observability matrix and, of course, result in strain estimates without the disturbance from the sixth natural frequency. On the other hand, further reduction of the modal basis would increase the modelling error in the design model.

It should be noted that the input forces are related to environmental conditions, turbulence and wave elevation, with a limited frequency content. Despite the nonlinear dependency of the actual forces on these conditions, the energy content of the higher frequencies is small. As a result, the structure mainly responds at its first and second natural frequency, and measurements at the tower only enable the local response estimation of the lattice structure. For normal environmental conditions, this restriction can be expected to be valid. For extreme conditions, for instance slamming waves during a storm, higher frequencies are excited, resulting in the dynamic response of local modes in the lattice structure. Since these modes are not observable by the adopted sensor network, the fatigue damage accumulated during these conditions cannot be estimated accurately. 
As a final remark it is mentioned that theoretically the sensor network could be reduced to one acceleration/velocity sensor and one displacement sensor. This network would not allow for the estimation of a separate equivalent wind and wave force, but it could be sufficient to estimate the response at unmeasured locations. The sensor locations, however, should be chosen such that the states relevant for the response are observed sufficiently.

\section{References}

[1] M. Muskulus and S. Schafhirt. Design optimization of wind turbine support structures - a review. Journal of Ocean and Wind Energy, 1(1):12-22, 2014.

[2] M.O.L. Hansen, J.N. Sørensen, S. Voutsinas, N. Sørensen, and H.A. Madsen. State of the art in wind turbine aerodynamics and aeroelasticity. Progress in Aerospace Sciences, 42(4):285-330, 2006.

[3] A.R. Henderson and M.B. Zaaijer. Hydrodynamic loading on offshore wind turbine support structures. Engineering Integrity, 25:24-31, 2014.

[4] R.J.S. Whitehouse, J.M. Harris, J. Sutherland, and J. Rees. The nature of scour development and scour protection at offshore windfarm foundations. Marine Pollution Bulletin, 62(1):73-88, 2011.

[5] M.L.A. Segeren. Influence of a boatlanding and J-tubes on wave loads and wall thickness of the monopile support structure design. In: Proceedings of the EWEA Offshore 2011 Conference, Amsterdam, The Netherlands, November 29 - December 12011.

[6] M.J. Kühn. Dynamics and Design Optimisation of Offshore Wind Energy Conversion Systems. PhD thesis, Delft University Wind Energy Research Institute, 2001.

[7] H. Hendrikse, F.W. Renting, and A.V. Metrikine. Analysis of the fatigue life of offshore wind turbine generators under combined ice- and aerodynamic loading. In: Proceedings of the ASME 2014 33rd International Conference on Ocean, Offshore and Arctic Engineering, San Francisco, California, USA, 2014.

[8] F. Vorpahl, M. Strobel, J.M. Jonkman, T.J. Larsen, P. Passon, and J. Nichols. Verification of aeroelastic offshore wind turbine design codes under IEA Wind Task XXIII. Wind Energy, 17(4):519-547, 2014.

[9] W.G. Versteijlen, K.N. van Dalen, A.V. Metrikine, and L. Hamre. Assessing the small-strain soil stiffness for offshore wind turbines based on in situ seismic measurements. Journal of Physics: Conference Series, 524(1), 2014.

[10] W.G. Versteijlen, A.V. Metrikine, J.S. Hoving, E. Smid, and W.E. de Vries. Estimation of the vibration decrement of an offshore wind turbine support structure caused by its interaction with soil. In: Proceedings of the EWEA Offshore 2011 Conference, Amsterdam, The Netherlands, November 29 December 12011.

[11] M.B. Zaaijer. Foundation modelling to assess dynamic behaviour of offshore wind turbines. Applied Ocean Research, 28:45-57, 2006.

[12] C.P. Fritzen, P. Kraemer, and M. Klinkov. An integrated SHM approach for offshore wind energy plants. In T. Proulx, editor, Proceedings of the IMAC-XXVIII, 727-740, Jacksonville, Florida, February $1-42010$.

[13] E. Lourens, C. Papadimitriou, S. Gillijns, S. Reynders, G. de Roeck, and G. Lombaert. Joint input-state estimation for structural systems based on reduced-order models and vibration data from a limited number of sensors. Mechanical Systems and Signal Processing, 29:310-327, 2012.

[14] C. Böker. Load Simulation and Local Dynamics of Support Structures for Offshore Wind Turbines. PhD thesis, Institute for Steel Construction, Leibniz Universität Hannover, 2009.

[15] W.E. de Vries, N.K. Vemula, P. Passon, T. Fischer, D Kaufer, D. Matha, B. Schmidt, and F. Vorpahl. Upwind wp4 d4.2.8 final report wp4.2: Support structure concepts for deep water. Technical report, UpWind, 2011.

[16] C. Papadimitriou, E. Lourens, G. Lombaert, G. de Roeck, and K. Liu. Prediction of fatigue damage accumulation in metallic structures by the estimation of strains from operational vibrations. In: LifeCycle and Sustainability of Civil Infrastructure Systems: Proceedings of the Third International Symposium on Life-Cycle Civil Engineering (IALCCE'12), Vienna, Austria, October 3-6, 2012, CRC Press, 2012.

[17] S. Gillijns and B. de Moor. Unbiased minimum-variance input and state estimation for linear discretetime systems with direct feedthrough. Automatica, 43(5):934-937, 2007.

[18] K. Maes, E. Lourens, K. van Nimmen, P. van den Broeck, P. Guillaume, G. de Roeck, and G. Lombaert. Verification of joint input-state estimation by in situ measurements on a footbridge. In F.-K. Chang, editor, $9^{\text {th }}$ International Workshop on Structural Health Monitoring, 1:343-350, Stanford, CA, USA, September 2013. 
[19] J. Jonkman, S. Butterfield, W. Musial, and Scott G. Definition of a 5-MW Reference Wind Turbine for Offshore System Development. Technical Report NREL/TP-500-38060, National Renewable Energy Laboratory, Golden, Colorado, February 2009.

[20] T. Burton, N. Jenkins, D. Sharpe, and E. Bossanyi. Wind Energy Handbook. Wiley, West Sussex, United Kingdom, second edition, 2011.

[21] M. Shinozuka and G. Deodatis. Simulation of stochastic processes by spectral representation. Applied Mechanics Reviews, 44(4):191--204, 1991.

[22] J.H. Vugts. Handbook of Bottom Founded Offshore Structures. Part 1. General Features of Offshore Structures and Theoretical Background. Eburon, Delft, The Netherlands, 2013.

[23] K. Maes, E. Lourens, K. van Nimmen, E. Reynders, G. de Roeck, and G. Lombaert. Design of sensor networks for instantaneous inversion of modally reduced order models in structural mechanics. Mechanical Systems and Signal Processing, 2014, http://dx.doi.org/10.1016/j.ymssp.2014.07.018i.

[24] E. Lourens. Force Identification in Structural Dynamics. PhD thesis, Katholieke Universiteit Leuven, 2012. 